\title{
Virtual Sensors: Using Data Mining Techniques to Efficiently Estimate Remote Sensing Spectra
}

\author{
Ashok N. Srivastava, Member, IEEE, Nikunj C. Oza, Member, IEEE, and Julienne Stroeve, Member, IEEE,
}

\begin{abstract}
Various instruments are used to create images of the Earth and other objects in the universe in a diverse set of wavelength bands with the aim of understanding natural phenomena. These instruments are sometimes built in a phased approach, with some measurement capabilities being added in later phases. In other cases, there may not be a planned increase in measurement capability, but technology may mature to the point that it offers new measurement capabilities that were not available before. In still other cases, detailed spectral measurements may be too costly to perform on a large sample. Thus, lower resolution instruments with lower associated cost may be used to take the majority of measurements. Higher resolution instruments, with a higher associated cost may be used to take only a smail fraction of the measuremenis in a given area. Many applied science questions that are relevant to the remote sensing commumity need to be addressed by analyzing enormous amounts of data that were generated from instruments with disparate measurement capability. This paper addresses this problem by demonstrating methods to produce high accuracy estimates of spectra with an associated measure of uncertainty from data that is perhaps nonlinearly correlated with the spectra. In particular, we demonstrate multi-layer perceptrons (MLPs), Support Vector Machines (SVMs) with Radial Basis Function (RBF) kernels, and SVMs with Mixture Density Mercer Kernels (MDMK). We call this type of an estimator a Virtual Sensor because it predicts, with a measure of uncertainty, unmeasured spectral phenomena.
\end{abstract}

Index Terms-Data Mining, Neural Networks, Support Vector Machine, Kernel Methods, Remote Sensing.

\section{INTRODUCTION}

$\mathbf{T}$ HIS paper describes the development of data mining algorithms that learn to estimate unobserved spectra from remote sensing data. For purposes of the discussion presented here, we will model the data as matrices of time series (following the notation in [1]). The spatiotemporal random function $Z(\mathbf{u}, \lambda, t)$ is modelled as a finite number $n$ of spatially correlated time series with the following representation:

$$
\begin{aligned}
Z(\mathbf{u}, \lambda, t) & =\left[Z_{\mathbf{u}}(\lambda, t)\right] \\
& =\frac{\left[Z_{u_{1}}(\lambda, t), Z_{u_{2}}(\lambda, t), \ldots, Z_{u_{n}}(\lambda, t)\right]^{T}}{}
\end{aligned}
$$

In Equation 1, $\mathrm{u}$ represents the spatial coordinate, $\lambda$ represents the vector of measured wavelength(s), and $t$ represents time. The superscript $T$ indicates the transpose operator. If multiple wavelengths are measured, then each $Z_{i}$ is actually a

Manuscript received March 15, 2004; revised November 18, 2002. This work was supported by the NASA Intelligent Systems Intelligent Data Understanding Program.

A. N. Srivastava and N. C. Oza are at the NASA Ames Research Center J. Stroeve is with the National Snow and Ice Data Center matrix, and the function $Z(\mathbf{u}, \lambda, t)$ represents a data cube of size $(n \times \Lambda \times T)$, where these symbols represent the number of spatial locations, the total number of measured wavelengths, and the total number of time samples, respectively. In this notation, the spatial coordinate $\mathbf{u}$ represents the coordinates (or index) of a measurement at a particular location in the field of view and is not in any way related to the distributed nature of the data centers. Conceptually, the equation above describes a set of $n(\Lambda \times T)$ matrices. In the event that the spatial coordinate describes adjacent pixels, it is useful to think of Equation 1 as describing a time series of data cubes (spectral images) of size $n \times n \times \dot{\Lambda}$.

Consider a situation where one is given a sensor $\mathcal{S}_{1}$ which takes $k$ spectral measurements in wavelength bands $\mathbf{B}_{1}=$ $\left\{\lambda_{1}, \lambda_{2}, \ldots, \lambda_{k}\right\}$ at time $t_{1}$. Suppose that we have another sensor $\mathcal{S}_{2}$ which has a set of spectral measurements taken at time $t_{2}, \mathbf{B}_{2}=\left\{\lambda_{1}, \lambda_{2}, \ldots, \lambda_{k}, \lambda_{k+1}, \lambda_{k+2}, \ldots, \lambda_{k+l}\right\}$ that partially overlaps the spectral features contained in $\boldsymbol{B}_{1}$ in terms of power in the spectral bands. Thus, $\mathbf{B}_{1}$ (or, in general, $\mathbf{B}_{1} \cap$ $\mathbf{B}_{2}$ ) are the common spectral measurements. Note that these measurements are common only in their power. $\mathbf{B}=\mathbf{B}_{\mathbf{2}} \backslash$ $\mathbf{B}_{1}=\left\{\lambda_{k+1}, \lambda_{k+2}, \ldots, \lambda_{k+l}\right\}$ represent the measurements available in $\mathbf{B}_{2}$ that are not available in $\mathbf{B}_{1}$. In this situation, we investigate the problem of building an estimator $\Gamma(Z(\mathbf{B}))$ that best approximates the joint distribution $P\left(Z(\mathbf{B}) \mid Z\left(\mathbf{B}_{1}\right)\right)$. Thus, we would have:

$$
\Gamma(Z(\mathbf{B})) \approx P\left(Z(\mathbf{B}) \mid Z\left(\mathbf{B}_{1}\right)\right)
$$

The value of building an estimator for $P$ is clear particularly in situations where one sensor has been in operation for a much longer period of time than another. The first sensor may have fewer spectral channels in which measurements are taken compared to the newer sensor. However, it may be of scientific value to be able to estimate what the spectral measurements in wavelengths $\mathbf{B}$ would have been had the first sensor had the measurement capability.

The joint distribution given by $P\left(Z(\mathbf{B}) \mid Z\left(\mathbf{B}_{1}\right)\right)$ above contains all necessary information to recover the underlying structure captured by the sensor $\mathcal{S}_{2}$. If perfect reconstruction of this joint distribution were possible, we would no longer need sensor $\mathcal{S}_{2}$ because all relevant information could be generated from the smaller subset of spectral measurements $B_{1}$ and the estimator $\Gamma$. Of course, such estimation is often extremely difficult because there is not sufficient information in the bands $\mathbf{B}_{1}$ to perfectly reconstruct the distribution. Also, in many cases, the joint distribution cannot be modelled properly using parametric representations of the probability distribution since 


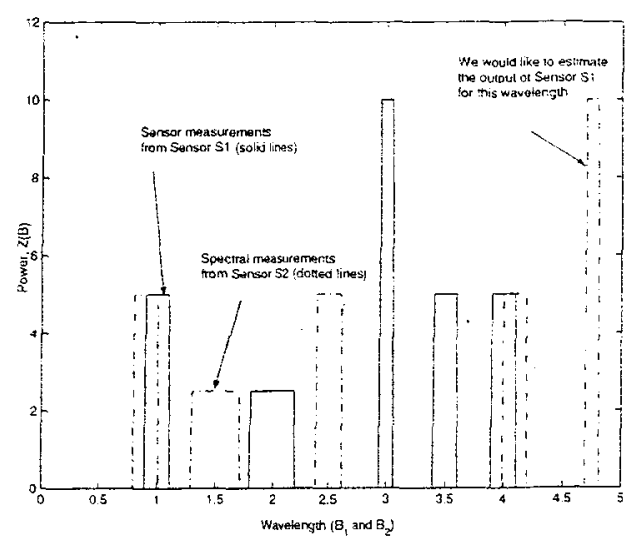

Fig. 1. This figure helps illustrate the need for a Virtual Sensor. We have spectral measurements from two sensors $\mathcal{S}_{1}$ and $\mathcal{S}_{2}$, (solid and dotted lines, respectively). We wish to estimate the output of sensor $\mathcal{S}_{1}$ for a wavelength where there is no actual measurement from the sensor. Note that some sensor measurements overlap perfectly, as in the case of wavelength $=3$, and in other cases, such as wavelength $=1$, there is some overlap in the measurements.

that may require a significant amount of domain knowledge and may be a function of the ground cover, climate, sun position, time of year, and numerous other factors.

In this paper, we describe methods to estimate the first moments of this distribution. Some methods that we use allow us to model the second moment of the distribution as well:

$$
\begin{aligned}
\mu(\mathbf{B}) & =\int \Gamma(\mathbf{B}) \mathrm{B} d \mathbf{B} \\
\sigma^{2}(\mathbf{B}) & =\int[\Gamma(\mathbf{B})-\mu(\mathbf{B})]^{2} \mathbf{B} d \mathbf{B}
\end{aligned}
$$

We use the function $\Gamma$ in the above computations as an estimate of the (unknown) joint distribution $P$. Several computational problems as well as problems due to the underlying physical measurement process arise when we attempt to estimate $\Gamma$. We begin by describing some of the problems that may arise due to the physical aspects of the two measurement devices and then discuss computational considerations.

Figure 1 gives a schematic view of the problem. The solid and dotted lines correspond to sensors $\mathcal{S}_{1}$ and $\mathcal{S}_{2}$ respectively. A Virtual Sensor can be built when there are some overlapping sensor measurements as depicted in the figure. Notice that if there are no overlapping sensor measurements, we are unable to build an estimator. In real-world problems, some measurements may overlap perfectly, while others have a partial overlap. Generally speaking the measurements from Sensor $\mathcal{S}_{1}$ are not available at all wavelength locations.

In the event that the spectral measurements are perfectly overlapping for all $k$ wavelength bands and the measurements for sensor $\mathcal{S}_{1}$ are not available at the remaining $\mathrm{B}$ bands, the estimation process is more straightfor ward. When partial overlap occurs between two sensors for a given wavelength, calculations need to be performed to estimate the amount of power that would have been measured in the overlapping bands. This can be done using interpolation methods.

Situations such as this often arise in practice. For example consider the relationship between the AVHRR (Advanced Very High Resolution Radiometer) and the MODIS (Moderate Resolution Imaging Spectroradiometer) instruments. Specifically, we show how to create a so-called Virtual Sensor to model
MODIS Channel 6 as a function of other MODIS channels that are also available in AVHRR. This way, the created model can be used to construct the virtual AVHRR channel 6 as a function of the other channels available in AVHRR. In our research, data mining methods are tested and their results examined for this task. New data mining algorithms are developed based on these results. Because of the large amounts of AVHRR and MODIS data available, we are focusing our development on producing high-quality results efficiently and quickly with principled estimates of uncertainty. Clearly, the construction of a Virtual Sensor has two key components. The first is constructing the model that generates the Virtual Sensor data given the known data. This requires training data-data for which there are true sensor values corresponding to the values of the Virtual Sensor. In this example, we would use MODIS images to generate a model that predicts MODIS channel 6 as a function of the other MODIS channels that are also available in AVHRR. Only channels common to MODIS and AVHRR can be used because of the second component of virtual sensor construction: generating the virtual sensor values. The learned model has to be used to generate the AVHRR virtual channel 6 as a function of the other AVHRR channels.

Some preliminary studies were made to check the feasibility of the Virtual Sensor using some MODIS and AVHRR images acquired over the Greenland ice sheet. In particular, supervised learning methods (e.g., neural networks) are capable of using MODIS data to construct a model that can predict MODIS channel 6 as a function of other MODIS channels. This model can then take an AVHRR image as input and can construct the virtual channel 6 .

\section{Virtual SENSORS For CRyosphere ANALysis}

Intensification of global warming in recent decades has caused a rise of interest in year-to-year and decadal-scale climate variability in the Polar Regions. This is because these regions are believed to be among the most sensitive and vulnerable regions to climatic changes. The enhanced vulnerability of the Polar Regions is believed to result from several positive feedbacks, including the temperature-albedomelt feedback and the cloud-radiation feedback. Recent observations of record regional anomalies in ice extent, thinning of the margins of the Greenland ice sheet, and reduction in the northern hemispheric snow cover, may reflect the effect of these feedbacks. Remote sensing products now provide spatially and temporally continuous and consistent information on several polar geophysical variables over nearly three decades. This period is sufficiently long enough to permit evaluation of how several cryospheric variables change in phase-with each other and with the atmosphere and can help to improve our understanding of the processes in the coupled land-ice-oceanatmosphere climate system. Cloud detection particularly over snow- and ice-covered surfaces is difficult using sensors such as AVHRR. This is because of the lack of spectral contrast between clouds and snow in the channels flown on the earlier AVHRR/2 sensors. Snow and clouds are both highly reflective in the visible wavelengths and often show little contrast in the thermal infrared. 
The AVHRR Polar Pathfinder Product (APP) consists of twice daily gridded (at 1.25 and $5 \mathrm{~km}$ spatial resolution) surface albedo and temperature from 1981 to 2000. A cloud mask accompanies this product but has been found to be inadequate. particularly over the ice sheets [5]. The 1.6 micron channel on the MODIS instrument as well as the AVHRR/3 sensor can significantly improve the ability to detect clouds over snow and ice. Therefore, by developing a virtual sensor to model the MODIS 1.6 micron channel as a function of the AVHRR/2 channels, we can improve the cloud mask in the APP product, and subsequently improve the retrievals of surface temperature and albedo in the product. In doing so we will be able to improve the accuracy in documenting seasonal and interannual variations in snow, ice sheet and sea ice conditions since 1981 .

\section{Creating a Virtual Sensor}

In this section we outline the procedure for creating a Virtual Sensor. At a minimum, we assume that for sensor $\mathcal{S}_{1}$ we have measurements $Z_{1}\left(\mathbf{B}_{1}\right)$ from one image, and for another sensor $\mathcal{S}_{2}$ we assume that we have another image $Z_{2}\left(\overline{\mathbf{B}}_{2}\right)$. The procedure for creating a Virtual Sensor is as follows, assuming that we need to build a predictor for channel $\mathbf{b}_{k+1}$ (recall that $k$ is the number of bands in $\mathbf{B}_{1}$ ):

1) Divide the data set $Z_{2}\left(\mathbf{B}_{2}\right)$ into a training set and a test set.

2) Find parameters $\theta$ that minimize the squared error (or another suitable metric) $\left[E\left[\Gamma\left(Z_{2}\left(\mathrm{~B}_{1}\right), \theta\right)\right]-Z_{2}\left(\mathbf{b}_{k+1}\right)\right]^{2}$.

3) Apply $\Gamma$ to the data from sensor $\mathcal{S}_{1}$ to generate an estimate of $E\left[\Gamma\left(Z_{1}\left(\mathbf{b}_{k+1}\right), \theta\right)\right]$. This is the step where the estimation of the unknown spectral contribution occurs.

4) Evaluate the results based on science based metrics and other information known about the image.

The procedure described above is standard in the data mining literature. From the remote sensing perspective, it is interesting to see the potentially systematic differences between the performance of the estimator on data from sensors 1 and 2 .

Note that this procedure will only work if sufficient information exists to predict $Z(\mathrm{~B})$ given data $Z\left(\mathrm{~B}_{1}\right)$. One simple procedure for determining this is to look at the linear correlation between the spectra. The top panel of Figure 2 shows the inter-channel linear correlation for the first seven channels of MODIS data. Larger squares indicate stronger linear correlation. Red squares indicate negative correlation and green squares indicate positive correlation. The lower panel in this figure shows the results of computing the mutual information between the pairs of channels. The mutual information between two random variables is given by:

$$
I(x, y)=\sum_{i=1}^{N} \sum_{j=1}^{M} P\left(x_{i}, y_{j}\right) \log \frac{P\left(x_{i}, y_{j}\right)}{P\left(x_{i}\right) P\left(y_{j}\right)}
$$
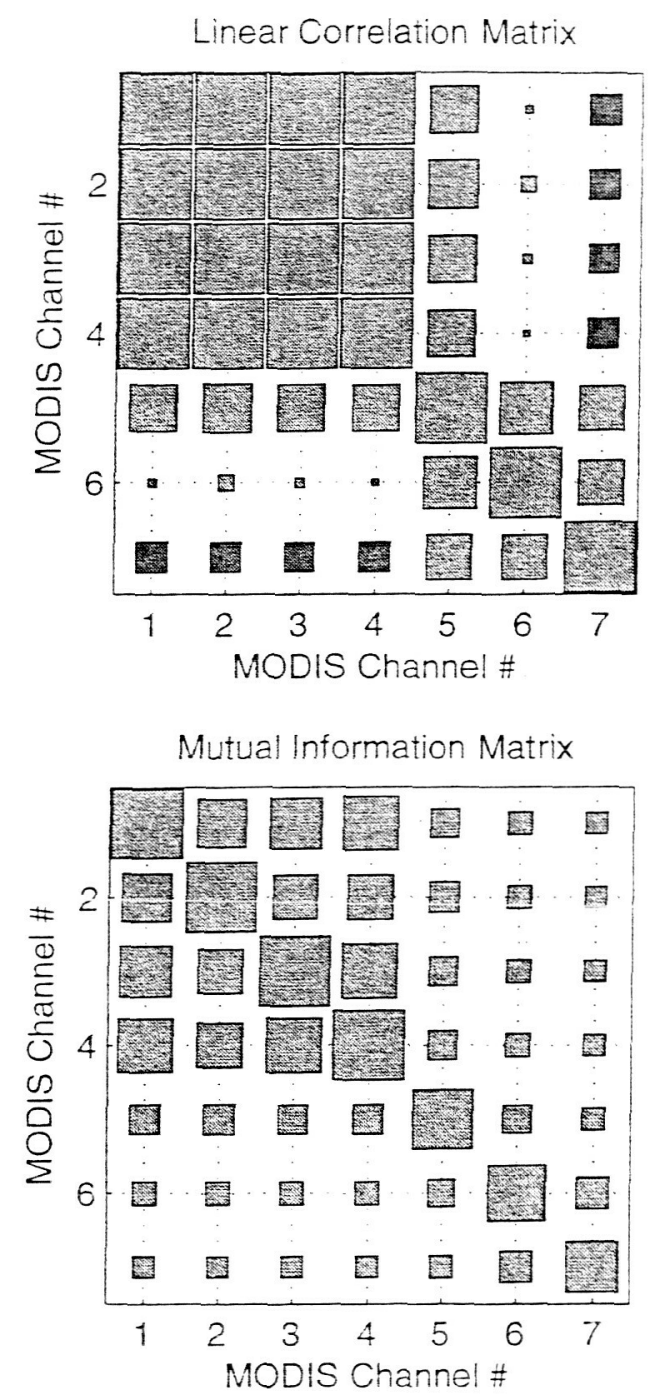

Fig. 2. The upper panel of this figure shows the linear correlation between the first seven channels of the MODIS instrument for one point in time. The size of the square indicates the degree of linear correlation. Green color indicates a positive correlation, and red color indicates a negative correlation. Notice that Channel 6 , which is a channel that we will ty to emulate using a Virtul Sensor, has a relatively weak correlation with the other channels. The lower panel indicates the mutual information between the same MODIS channels. Notice that for this nonlinear measure of information, channel 6 has more relationship with the other channels, thus giving hope that a nonlinear model could be built to predict channel 6 .

This method gives a nonlinear measure of the relationship between the channels. Again, the larger the square, the greater the degree of relationship. In the case described in this paper, we will be building models in order to predict Channel 6 . Notice that Channel 6 has small linear correlations with the other channels but moderate mutual information.

The next section describes three estimation methods that we have used to build a Virtual Sensor: a feed-forward neural network (also called a multilayer perceptron, (MLP)), a Support Vector Machine (SVM), and an SVM with a Mixture Density Mercer Kernel.

\section{Standard Data Mining Methods}

There are many machine learning methods that have been used in many different types of problems. We give a brief 


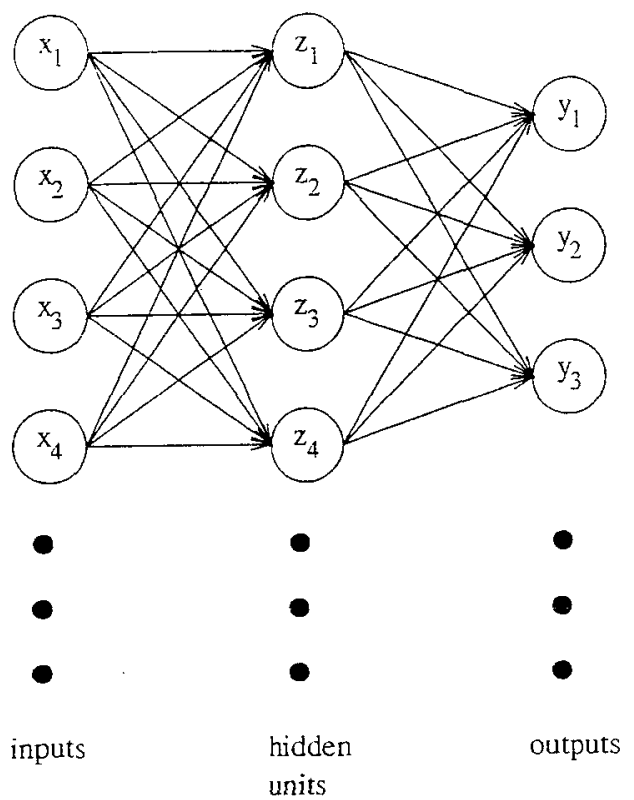

Fig. 3. An example of a Multilayer Percepron (MLP).

review of the methods that we use in this paper.

\section{A. Multi-Layer Perceptrons}

We first describe multilayer perceptrons, a type of neural network [4]. The central idea of neural networks is to construct linear combinations of the inputs as derived features, and then model the target as a nonlinear function of these derived features. Neural networks are often depicted as a directed graph consisting of nodes and arcs. An example is shown in Figure 3. Each column of nodes is a layer. The leftmost layer is the input layer. The inputs of an example to be classified are entered into the input layer. The second layer is the hidden layer and the third layer is the output layer. Information flows from the input layer to the hidden layer and then to the output layer via a set of arcs (depicted in figure 3 as arrows). Note that the nodes within a layer are not directly connected. In our example, every node in one layer is connected to every node in the next layer, but this is not required in general. Also, a neural network can have more or less than one hidden layer and can have any number of nodes in each hidden layer.

Each non-input node, its incoming arcs, and its single outgoing arc constitute a neuron, which is the basic computational element of a neural network. Each incoming arc multiplies the value coming from its origin node by the weight assigned to that arc and sends the result to the destination node. The destination node adds the values presented to it by all the incoming arcs, transforms it with a nonlinear activation function (to be described later), and then sends the result along the outgoing arc. For example, the return value of a hidden node $z_{j}$ in our example neural network is

$$
z_{j}=g\left(\sum_{i=1}^{|A|} w_{i, j}^{(1)} x_{i}\right),
$$

where $|A|$ is the number of input units, $w_{i, j}^{(k)}$ is the weight on the arc in the $k$ th layer of arcs that goes from unit $i$ in the $k$ th layer of nodes to unit $j$ in the next layer (so $w_{i, j}^{(1)}$ is the weight on the arc that goes from input unit $i$ to hidden unit $j$ ) and $g$ is a nonlinear activation function. A commonly used activation function is the sigmoid function:

$$
g(a) \equiv \frac{1}{1+\exp (-a)} .
$$

The return value of an output node $y_{j}$ is

$$
y_{j}=g\left(\sum_{i=1}^{Z} w_{i, j}^{(2)} z_{i}\right)
$$

where $Z$ is the number of hidden units. The outputs are clearly nonlinear functions of the inputs.

Neural networks are trained to fit data by a process that is essentially nonlinear regression. Given each entry in the training dataset, the network's current prediction is calculated. The difference between the true function value and the prediction is the error. The derivative of this error with respect to each weight in the network is calculated and the weights are adjusted accordingly to reduce the error.

\section{B. Support Vector Machines}

Support Vector Machines for classification and regression are described in detail in [3], but here we briefly describe Support Vector Regression (SVR), which we use in this paper. In real-world problems, traditional linear regression cannot be expected to fit a set of points perfectly (i.e., with zero error). For this reason, nonlinear regression is often used with the hope that a more powerful nonlinear model will achieve a better fit than a linear model. However, this power often comes with two drawbacks. One is that the space of parameters of a nonlinear model (such as the multilayer perceptrons discussed above) often have many local optima that are not globally optimal. Nonlinear regression algorithms such as backpropagation for MLPs often find these local optima, which can result in a model that does not predict well on unseen data. The second drawback is that nonlinear model fitting is often overly sensitive to the locations of the training points, so that they overfit the training points and do not perform well on new data.

SVR addresses these problems in three ways. The first way is to use an $\epsilon$-insensitive loss function. If $y$ is the true response and $f(\mathbf{x})$ is the predicted response for the input $\mathbf{x}$, then the loss function is

$$
|y-f(\mathbf{x})|_{\epsilon}=\max \{0,|y-f(\bar{x})|=\bar{\epsilon}\}
$$

That is, if the error between the true response and the predicted response is less than some small $\epsilon$, then the error on that point is considered to be zero. For example, in figure 4 , the solid line, which is the fitted line, is within $\epsilon$ of all the points between the two dashed lines; therefore, the error is considered to be zero for those points. If $\epsilon$ is set to the level of the typical noise that one can expect in the response variable, then support vector regression is less likely to expend effort fitting 


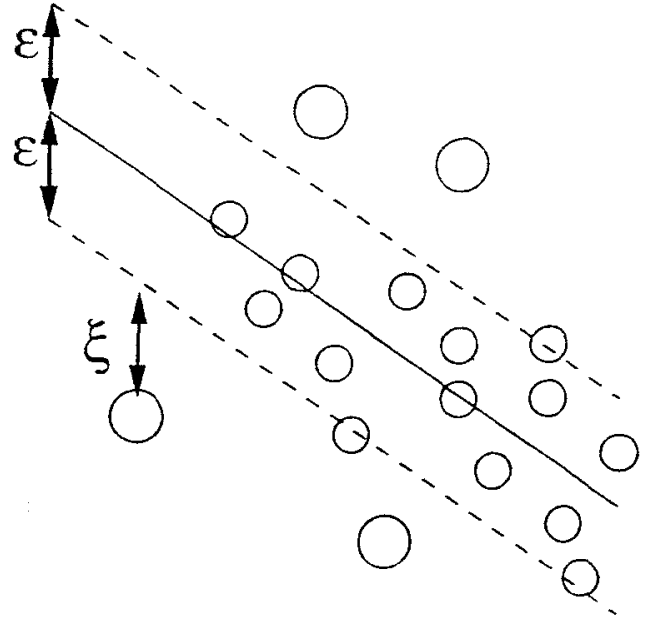

Fig. 4. Support Vector Machine for regression. The solid line is the line fitted to the points (represented as circles). The dashed lines are a distance $\epsilon$ from the fitted line. The points within the dashed line are considered to have zero error by an $\epsilon$-insensitive loss function.

the noise in the training data at the expense of generalization performance, i.e., it is less likely to overfit. In particular, to estimate the linear regression

$$
f(\mathbf{x})=\mathbf{w} \cdot \mathbf{x}+b
$$

one solves the optimization problem of minimizing

$$
\frac{1}{2}\|\mathbf{w}\|^{2}+C \sum_{i=1}^{m}\left|y_{i}-f\left(\mathbf{x}_{i}\right)\right|_{\epsilon},
$$

where $C$ is a user-determined constant that determines the tradeoff between the closeness of the fit (second term) and the level of regularization (first term).

The second way support vector regression addresses the above problems is to allow some error beyond $\epsilon$ for each training point but minimize the total such error over all the points. In figure $4, \xi$ is the additional error for one particular point. For $m$ training points, define $\xi_{i}$ for $i \in\{1,2, \ldots, m\}$ to be the slack variables that represent the additional allowable error if $f\left(\mathbf{x}_{i}\right)-y_{i}>\epsilon$ (i.e., $\xi_{i}=0$ otherwise) and $\xi_{i}^{*}$ to be the additional error if $y_{i}-f\left(\mathbf{x}_{i}\right)>\epsilon$. In that case, the optimization problem is the following:

$$
\begin{aligned}
\operatorname{minimize} \frac{1}{2}\|\mathbf{w}\|^{2} & +C \sum_{i=1}^{m}\left(\xi_{i}+\xi_{i}^{*}\right) \\
\text { subject to } & \\
f\left(\mathbf{x}_{i}\right)-y_{i} & \leq \epsilon+\xi_{i} \\
y_{i}-f\left(\mathbf{x}_{i}\right) & \leq \epsilon+\xi_{i}^{*} \\
\xi_{i} & \geq 0 \\
\xi_{i}^{*} & \geq 0
\end{aligned}
$$

for all $i \in 1,2, \ldots, m$.

Note that the above optimization problem will involve minimizing the sum of the slack variables. Also, any points for which the error is already less than $\epsilon$ will end up with zero for their corresponding slack variables. Because this is a convex optimization problem, there is a unique globally optimal solution.

The third way that SVR addresses the above problems is to map the data from the original data space into a much higher (possible infinite) dimensional feature space and calculate the support vector machine in that space. The idea is that the linear model in the feature space may correspond to a complicated nonlinear model in the original data space. Clearly, one needs a practical way to deal with data that is mapped to such a high-dimensional space, which intuitively seems impossible. However, one is able to do this using the kernel trick. By introducing Lagrange multipliers and obtaining the dual of the previous optimization problem (see [3] for the details), one obtains the following:

$$
\begin{array}{r}
\operatorname{maximize}_{\alpha, \alpha^{*} \in \mathcal{R}}-\epsilon \sum_{i=1}^{m}\left(\alpha_{i}^{*}+\alpha_{i}\right)+\sum_{i=1}^{m}\left(\alpha_{i}^{*}-\alpha_{i}\right) y_{i} \\
-\frac{1}{2} \sum_{i=1}^{m} \sum_{j=1}^{m}\left(\alpha_{i}^{*}-\alpha_{i}\right)\left(\alpha_{j}^{*}-\alpha_{j}\right) \mathbf{x}_{i} \cdot \mathbf{x}_{j} \\
\text { subject to } 0 \leq \alpha_{i}, \alpha_{i}^{*} \leq C \text { for all } i \in\{1,2, \ldots, m\} \\
\text { and } \sum_{i=1}^{m}\left(\alpha_{i}-\alpha_{i}^{*}\right)=0 .
\end{array}
$$

The resulting regression estimate is of the form

$$
f(\mathbf{x})=\sum_{i=1}^{m}\left(\alpha_{i}^{*}-\alpha_{i}\right) \mathbf{x}_{i} \cdot \mathbf{x}_{j}+b .
$$

Note that the inputs only appear as dot products in the above solution. Therefore, one can map the inputs into a very high or even infinite dimensional space $H$ using a function $\Phi: \mathcal{R}^{d} \rightarrow$ $H$ and the dot product $\Phi\left(\mathbf{x}_{i}\right) \cdot \Phi\left(\mathbf{x}_{j}\right)$ will still be a scalar. Of course, $\Phi$ would be too difficult to work with because of the high dimensionality of $H$. However, there exist kernel functions $K\left(x_{i}, x_{j}\right)=\Phi\left(\mathbf{x}_{i}\right) \cdot \Phi\left(\mathbf{x}_{j}\right)$ such that $K$ is practical to work with even though the $\Phi$ induced by that $K$ is not. For example, the Gaussian kernel

$$
K\left(\mathbf{x}_{i}, \mathbf{x}_{j}\right)=e^{\frac{-\left\|x_{i}-x_{j}\right\|}{2 \sigma^{2}}}
$$

gives rise to a $\Phi$ that is infinite-dimensional. However, we do not need to deal with $\Phi$ or even know what it is because the $\Phi$ 's only appear as dot products, which can be replaced by $K$. Therefore, the new regression estimate after mapping the inputs from the data space to the feature space is

$$
f(\mathbf{x})=\sum_{i=1}^{m}\left(\alpha_{i}^{*}-\alpha_{i}\right) K\left(\mathbf{x}_{i}, \mathbf{x}_{j}\right)+b .
$$

In summary, the Support Vector Machine allows us to fit a nonlinear model to data without the local optima problem that other procedures suffer from.

The kernel function can be viewed as a measure of similarity between two data points. For example, with the Gaussian 
kernel, the value increases as the distance between the pair of points decreases. There is significant current research attempting to determine which kernel functions are most appropriate for different types of problems. One such novel kernel function is the Mixture Density Mercel Kernel (MDMK) which is discussed in the next section.

\section{Mixture Density Mercer Kermels}

The idea of using probabilistic kernels was discussed by Haussler in 1999 [2] where he observes that if $K\left(\mathbf{x}_{i}, \mathbf{x}_{j}\right) \geq 0$ $\forall\left(\mathbf{x}_{i}, \mathbf{x}_{j}\right) \in \mathcal{X} \times \mathcal{X}$, and $\sum_{\mathbf{x}_{i}} \sum_{\mathbf{x}_{j}} K\left(\mathbf{x}_{i}, \mathbf{x}_{j}\right)=1$ then $K$ is a probability distribution and is called a P-Kernel. He further observed that the Gibbs kernel $K\left(\mathrm{x}_{i}, \mathrm{x}_{j}\right)=P\left(\mathrm{x}_{i}\right) P\left(\mathrm{x}_{j}\right)$ is also an admissible kernel function.

Our idea is to use an ensemble of probabilistic mixture models as a similarity measure. Two data points will have a larger similarity if multiple models agree that they should be placed in the same cluster or mode of the distribution. Those points where there is disagreement will be given intermediate similarity measures. The shapes of the underlying mixture distributions can significantly affect the similarity measurement of the two points. Experimental results uphold this intuition and show that in regions where there is "no question" about the membership of two points, the Mixture Density Kernel behaves identically to a standard mixture model. However, in regions of the input space where there is disagreement about the membership of two points, the behavior may be quite different than the standard model. Since each mixture density model in the ensemble can be encoded with domain knowledge by constructing informative priors, the Mixture Density Mercer Kernel (MDMK) will also encode domain knowledge. The MDMK is defined as follows:

$$
\begin{aligned}
K\left(\mathbf{x}_{i}, \mathbf{x}_{j}\right) & =\Phi^{T}\left(\mathbf{x}_{i}\right) \Phi\left(\mathbf{x}_{j}\right) \\
\cdot & =\frac{1}{Z\left(\mathbf{x}_{i}, \mathbf{x}_{j}\right)} \sum_{m=1}^{M} \sum_{c_{m}=1}^{C_{m}} P_{m}\left(c_{m} \mid \mathbf{x}_{i}\right) P_{m}\left(c_{m} \mid \mathbf{x}_{j}\right)
\end{aligned}
$$

The feature space is thus defined explicitly as follows:

$$
\begin{aligned}
\Phi\left(\mathbf{x}_{i}\right) \propto & {\left[P_{1}\left(c=1 \mid \mathbf{x}_{i}\right), P_{1}\left(c=2 \mid \mathbf{x}_{i}\right), \ldots,\right.} \\
& \left.P_{1}\left(c=C \mid \mathbf{x}_{i}\right), P_{2}\left(c=1 \mid \mathbf{x}_{i}\right), \ldots, P_{M}\left(c=C \mid \mathbf{x}_{i}\right)\right]
\end{aligned}
$$

The first sum in the defining equation above sweeps through the $M$ models in the ensemble, where each mixture model is a Maximum A Posteriori estimator of the underlying density trained by sampling (with replacement) the original data. We will discuss how to design these estimators in the next section. $C_{m}$ defines the number of mixtures in the $m$ th ensemble, and $c_{m}$ is the cluster (or mode) label assigned by the model. The quantity $Z\left(\mathbf{x}_{i}, \mathbf{x}_{j}\right)$ is a normalization such that $K\left(\mathbf{x}_{i}, \mathbf{x}_{i}\right)=1$ for all $i$. The fact that the Mixture Density Kernel is a valid kernel function arises directly from the definition.

The Mixture Density Kernel function can be interpreted as follows. Suppose that we have a hard classification strategy, where each data point is assigned to the most likely posterior class distribution. In this case the kernel function counts the the number of times the $M$ mixtures agree that two points should be placed in the same cluster mode. In soft classification,

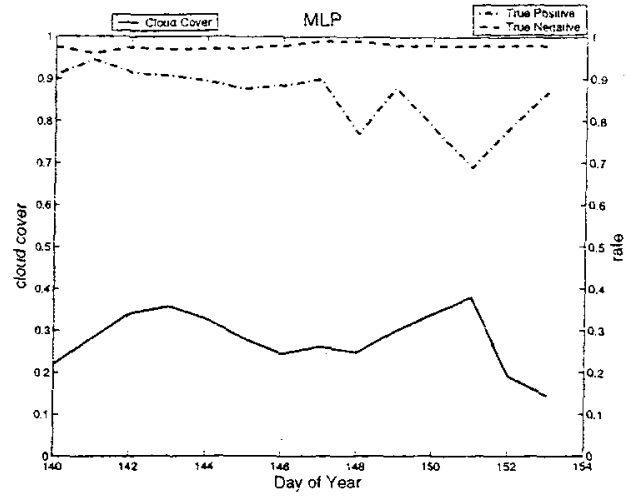

Fig. 5. MODIS predictions from year 2000, days 140-153. This image shows the percent cloud cover in each image as well as the true positive and true negative rates for MLPs on these images.

two data points are given an intermediate level of similarity (between 0 and 1) which will be less than or equal to the case where all models agree on their membership, in which case the entry would be unity. Further interpretation of the kernel function is possible by applying Bayes rule to the defining equation of the Mixture Density Kernel. Thus, we have:

$$
\begin{aligned}
K\left(\mathbf{x}_{i}, \mathbf{x}_{j}\right)= & \frac{1}{Z\left(\mathbf{x}_{i}, \mathbf{x}_{j}\right)} \sum_{m=1}^{M} \sum_{c_{m}=1}^{C_{m}} \frac{P_{m}\left(\mathbf{x}_{i} \mid c_{m}\right) P_{m}\left(c_{m}\right)}{P_{m}\left(\mathbf{x}_{i}\right)} \times \\
& \frac{P_{m}\left(\mathbf{x}_{j} \mid c_{m}\right) P_{m}\left(c_{m}\right)}{P_{m}\left(\mathbf{x}_{j}\right)} \\
= & \frac{1}{Z\left(\mathbf{x}_{i}, \mathbf{x}_{j}\right)} \sum_{m=1}^{M} \sum_{c_{m}=1}^{C_{m}} \frac{P_{m}\left(\mathbf{x}_{i}, \mathbf{x}_{j} \mid c_{m}\right) P_{m}^{2}\left(c_{m}\right)}{P_{m}\left(\mathbf{x}_{i}, \mathbf{x}_{j}\right)}
\end{aligned}
$$

The second step above is valid under the assumption that the two data points are independent and identically distributed. This equation shows that the Mixture Density Kernel measures the ratio of the probability that two points arise from the same mode, compared with the unconditional joint distribution. If we simplify this equation further by assuming that the class distributions are uniform, the kernel tells us on average (across ensembles) the amount of information gained by knowing that two points are drawn from the same mode in a mixture density.

\section{RESULTS}

To derive the results described in this section, we trained the three methods described above on a small subset of a MODIS image from day 140 . We first selected those pixels for which channel 1 was at least 0.3 , thereby removing pixels that are over open water and keeping the snow/ice-covered areas. This turned out to be about half of this particular image (1.6 million pixels). Out of these pixels, we chose about 2500 of them at random for training. In all cases, the inputs were the five MODIS channels that correspond most closely to the five AVHRR/2 channels (see the Appendix for tables with AVHRR/2 and MODIS instrument specifications). That is, the inputs were channels $1,2,20,31$, and 32 . The output to be predicted was channel 6 .

\section{A. MODIS Results}

In testing the MODIS channel 6 prediction algorithms, 13 MODIS images from the year 2000 were processed. All the 


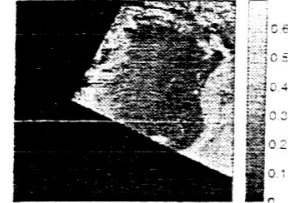

MODIS channel 6

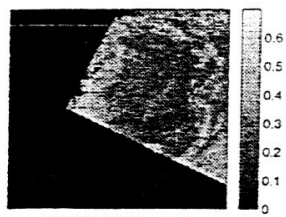

SVM REF kernel

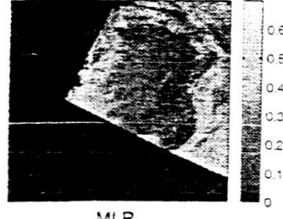

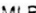

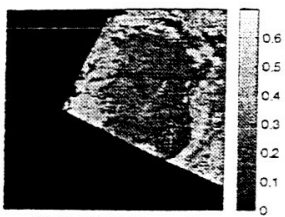

SVM MDMK kerne
Fig. 6. MODIS predictions from year 2000 , day 149. (a) Upper Left. Channe! 1. (b). Upper Right. Prediction of an MLP. (c) Lower Left. Prediction of an SVM with RBF kernel. (d). Lower Right. Prediction of an SVM with MDMK kernel. The black areas with straight boundaries are regions containing no data.

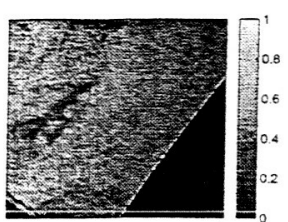

AVHFR channel 1

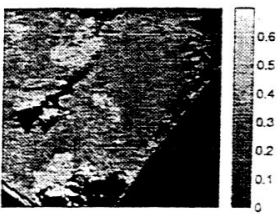

SVIM ABF kerne
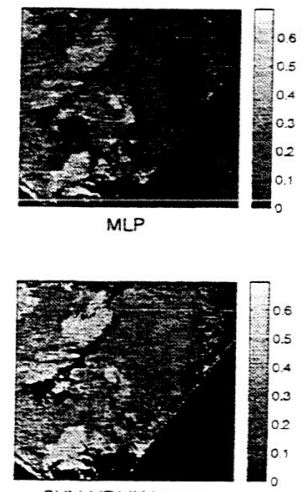

SVM MDMK kerne
Fig. 7. AVHRR predictions from year 2000, day 140. (a) Upper Left. Channel 1. (b). Upner Right. Prediction of an MLP. (c) Lower Left. Prediction of an SVM with RBF kernel. (d). Lower Right. Prediction of an SVM with MDMK kernel. The black areas with straight boundaries are regions containing no data.

MODIS imagery were gridded to a $1.25 \mathrm{~km}$ Equal Area Scalable Earth Grid (EASE-Grid) [6]). Figure 5 summarizes the amount of cloud cover for each day (defined using a threshold of 0.3 on the MODIS channel 6 images) together with the true positive and true negative cloud cover retrieval rates by the MLP model. The true positive retrieval rate is defined as the number pixels predicted to have cloud cover that actually have cloud cover divided by the total number of pixels that actually have cloud cover. Similarly, the true negative retrieval rate is the number of pixels predicted to not have cloud cover that actually do not have could cover divided by the total number of pixels that actually do not have cloud cover. The MLP appears to accurately model areas that are of low reflectance in the MODIS channel 6 (e.g. no clouds) as seen by the high rate of true negative retrieval. The MLP model is slightly less successful in always correctly modelling the high reflectance (e.g. clouds), but the overall positive retrieval rate is still relatively high (70 to $90 \%)$.

\section{B. AVHRR REsults}

We now discuss our results on two AVHRR images. Figure 7 (a) shows the visible (channel 1) top-of-the-atmosphere (TOA) reffectance from AVHRR for day 140 over the Green-
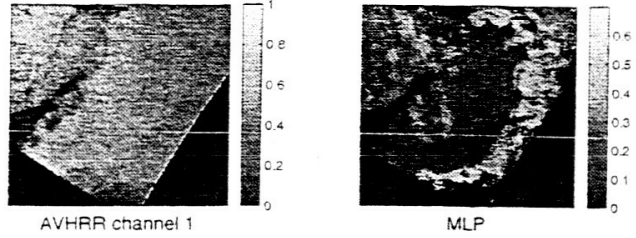

MLP
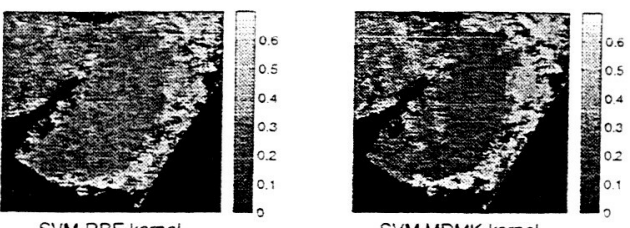

SVM MDMK kernel

Fig. 8. AVHRR predictions from year 2000, day 150. (a) Upper Left. Channel 1. (b). Upper Right. Prediction of an MLP. (c) Lower Left. Prediction of an SVM with RBF kemel. (d). Lower Right. Prediction of an SVM with MDMK kernel. The black areas with straight boundaries are regions containing no data.

land ice sheet. Note also that not only is the ice sheet shown in the image, but also open water areas (black color) and sea ice. Clouds are evident by textural variations in the south, central and northwestern part of the ice sheet. Some clouds also appear brighter and some darker than the underlying snow. In Figure 7 (b) through (d) the TOA reflectance predictions for a channel at 1.6 microns are shown. The MLP prediction (Figure 7(b)) indicates that the majority of the ice sheet is cloud free (very low reflectance at 1.6 microns) and the clouds that are seen as textural variations in the visible image show up as bright (higher reflectance) in the image. Most of the clouds are captured, although the few scattered clouds in the northwest part of the ice sheet are not detected. In addition, some of the clouds in the central part of the ice sheet may be classified as "clear" if a threshold of 0.3 is selected. A more conservative threshold may be 0.2 , which is currently used by the MODIS snow cover team to discriminate between clouds and snow. The SVM RBF (Figure 7(c)) picks up the clouds as well (the brighter areas in the image), but this method also starts to distinguish between different snow types as evident by the slightly different reflectance values along the western margin of the ice sheet. Further discrimination of different snow types is observed in the SVM MDMK (Figure 7(d)) image. Note however, in both of these models, picking a threshold of 0.3 to decide whether or not the pixel is clear or cloudy may cause areas of the ice sheet that are actually clear to be labeled as clouds. Thus, additional information would be needed in order to distinguish between atmospheric variations (i.e. clouds) and variations in the snow/ice conditions. Note also that, off the northwestern coast of the ice sheet, areas that are sea ice with no cloud cover appear as clouds (higher reflectance) in the prediction models.

Figure 8(a)-(d) shows the same results as discussed above but for day 150. The visible image (Figure 8(a)) suggests that the entire western margin of the ice sheet is cloudy as well as the north centraleastern part of the ice sheet. The MLP (Figure 8(b)) captures some of the scattered clouds along the western margin of the ice sheet, but misses quite a few of them, especially in the southern part. Similarly, in the northeastern part of the ice sheet, the MIP is not capturing 
all the clouds observed in the visible image. The SVM RBF (Figure 8(c)) model does a better job in detecting the clouds in the northeastern region of Greenland as well as along the west coast. The SVM MDMK model further detects some clouds that are missed in the SVM RBF model (e.g. along the southwest edge of Greenland) and also begins to highlight more of the different snow/ice types.

These two different examples help to illustrate that simulating a 1.6 micron sensor channel does not necessarily capture all the clouds. In general, snow has very low reflectance at 1.6 microns, whereas clouds have high reflectance. Thus, we would expect snow cover to be bright in the visible channel and dark in at 1.6 microns. However, cloud reflectance at 1.6 microns depends in part on the cloud type and may be bright or less bright (e.g. gray).

In the day 140 example, the MLP prediction does capture most all of the clouds observed in the visible image. For this day, the 1.6 micron is a good cloud classifier. On day 150 however, the MLP prediction does not perform quite as well. Even though it may still accurately predict the TOA reflectance at 1.6 microns, some clouds are missed.

\section{CONCLUSION}

In this paper we have presented the development of data mining algorithms to estimate unobserved spectra. We call this estimation "Virtual Sensors." We presented some results on a particular instantiation of Virtual Sensors: the estimation of MODIS channel 6 for AVHRR. Our motivation for choosing this particular problem is to aid in the discrimination of clouds from snow and ice. This is a challenging problem that is essential to solve in order to map the cryosphere using visible and thermal imagery. Clouds often have similar spectral reflectance as snow and similar temperatures. Most cloud detection algorithms operationally employ a series of spectral tests to determine if a pixel is clear or cloudy. Having a channel centered around 1.6 microns has significantly improved the ability to discriminate between clouds and snow using new sensors such as MODIS and AVHRR/3. Unfortunately, a vast amount of data have been collected before these sensors existed that did not have a channel designed to detect clouds over snow and ice-covered surfaces. These data sets have large importance for climate studies since they provide over 20 years worth of observations. Thus, being able to improve the cloud masking abilities of these previous sensors will allow for improved monitoring of several cryospheric variables, such as surface albedo, surface temperature, snow and ice cover.

In the above analysis, we used calibrated TOA reflectances from the MODIS and AVHRR instruments: These reffectance values are dependent upon the specific viewing and illumination geometry of the orbit considered. This may or may not lead to some errors since snow and clouds do not reflect the incoming solar radiation isotropically. The magnitude of this effect remains to be determined. However, given the robustness of the method, the angular variability of the reflectance may fall into the "noise" of the data so that our methods can be applied prior to using methods to correct for the angular variability of the TOA reffectance.
We plan to extend our work on the problem of estimating MODIS channel 6 for AVHRR images in several directions. In order to see how quickly our methods can learn a good quality model, we trained on very little data. We plan to train on additional data over different times of year to understand how much improvement is possible. We plan to develop more scalable algorithms that will allow us to train on large amounts of data in a practical amount of time. For example active learning algorithms only process examples on which the current model's predictions are significantly in error and do not waste effort on the remaining examples the way traditional machine learning algorithms do. Online learning algorithms process training examples only once rather than repeatedly cycling through them the way traditional algorithms do. We also plan to perform a more detailed analysis of the results over more images from different years and different times of year in order to better understand the situations in which different data mining algorithms are most effective. This may lead to the development of a hybrid scheme (ensemble) that performs better than any one method. Our algorithms currently only train on and generate predictions for individual pixels in individual images. Spatial correlation and temporal correlation will be accounted for in our future work.

We also plan to go beyond the particular problem of predicting channel 6 to predicting other channels and quantities that are of scientific importance. We will attempt to quantify cross-channel information through further mutual information studies.

\section{APPENDIX I \\ INSTRUMENT SPECIFICATIONS}

Tables II and II contain specifications of the AVHRR/2 and MODIS instruments, respectively.

TABLE I

AVHRR/2 INSTRUMENT SPECIFICATIONS

\begin{tabular}{|c|c|c|}
\hline Channel Number & Wavelength & Purpose \\
\hline 1 & 0.58 to 0.68 & $\begin{array}{c}\text { Cloud Cover } \\
\text { Snow Cover } \\
\text { Vegetation Index }\end{array}$ \\
\hline 2 & 0.725 to 1.00 & $\begin{array}{c}\text { Earth Radiation Budget } \\
\text { Surface Water Boundaries } \\
\text { Vegetation Index }\end{array}$ \\
\hline 3 & 3.55 to 3.93 & $\begin{array}{l}\text { Water Vapor Correction } \\
\text { Thermal Mapping }\end{array}$ \\
\hline 4 & 10.3 to 11.3 & Thermal Mapping \\
\hline 5 & 11.5 to 12.5 & $\begin{array}{l}\text { Water Vapor Correction } \\
\text { Thermal Mapping }\end{array}$ \\
\hline
\end{tabular}

\section{ACKNOWLEDGMENT}

The authors would like to thank Brett Zane-Ulman for preparation of the figures shown here and others used in our analysis, as well as many of the results presented.

\section{REFERENCES}

[1] P. C. Kyriakidis and A. G. Journel, "Geostatistical space-time models: A review," Mathematical Geology, vol. 31, no. 6, pp. 651-684, 1999.

[2] D. Haussler, "Convolution kernels on discrete structures," University of Califomia Santa Cruz, Tech. Rep., 1999. 
[3] B. Schölkopf and A. Smola, Leaming with Kernels. MIT Press, 2002.

[4] C.M. Bishop, Neural Networks for Pattem Recognition. Oxford University Press, 1995

[5] J. Stroeve, "Assessment of Greenland Albedo Variability from the AVHRR Polar Pathfinder Data Set," Journal of Geophysical ResearchAtmospheres, 106(D24), 33989-34005.

[6] R.L. Amstrong and M.J. Brodzik, "Earth-gridded SSM/ data set for cryospheric studies and global change monitoring," In A1 Symposium of COSPAR Scientific Commission A, Hamburg, Germany, July 11 21, 1994. Proceedings Satellite Monitoring of the Earth's Surface and Atmosphere, Nov 1995, 115-163.

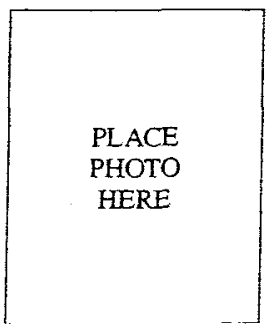

Ashok N. Srivastava Dr. Ashok N. Srivastava is a Principal Scientist and Group Leader in the Data Mining and Complex Adaptive Systems Group at NASA Ames Research Center. He has fourteen years of research, development, and consulting experience in machine leaming, data mining, and data analysis in time series analysis, signal processing, and applied physics. Dr. Srivastava has had significant experience both in research (NASA, NIST, IBM) as well as the business world at IBM (Senior Consultant) and Bilue Martuni Sottware (Senior Director).

Dr. Srivastava's machine learning research interests include topics in kernel methods, assessment of linear and nonlinear covariability, understanding and forecasting time-based data, and image processing. He is also interested in distributed data mining and scalability issues in federated data systems. A primary area of applied research is in the development of onboard satellite algorithms for automatic defecting and discovery of geophysical processes.

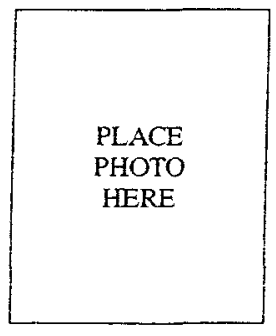

Nikunj C. Oza Dr. Nikunj C. Oza has been a Research Scientist at NASA Ames Research Center since September, 2001. He received his B.S. in Mathematics with Computer Science from the Massachusetts Institute of Technology (MIT) in 1994, and M.S. and Ph.D. in Computer Science from the University of California at Berkeley in 1998 and 2001, respectively. His research interests include ensemble learning, online learning, and applications of machine learning to such problems as fault detection and remote sensing.

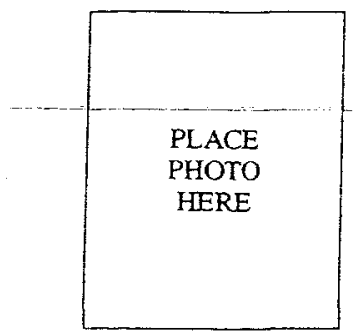

Julienne Stroeve Dr. Julienne C Stroeve has been a research scientist at the Narional Snow and Ice Data Center (NSIDC) since 1996. She received her B.S. (1989) and M.S. (1991) in Aerospace Engineering from the University of Colorado. Her Ph.D. was received in 1996 from the Geography Department at the University of Colorado where her thesis dealt with deriving a radiation climatology of the Greenland ice sheet using satellite imagery. Her research interests include optical, thermal and microwave remote sensing of snow and ice-covered surfaces, cryosphere climate interactions, atmospheric radiative transfer modeling, and image processing.
TABLE II

MODIS INSTRLMENT SPECIFCATIONS

\begin{tabular}{|c|c|c|}
\hline Band & Bandwidth & Primary Use \\
\hline 1 & $620-670$ & $\begin{array}{c}\text { Land/Cloud/Aerosols } \\
\text { Boundaries }\end{array}$ \\
\hline 2 & $841-876$ & $\begin{array}{c}\text { Land/Cloud/Aerosols } \\
\text { Boundaries }\end{array}$ \\
\hline 3 & $459-479$ & $\begin{array}{c}\text { Land/Cloud/Aerosols } \\
\text { Properties }\end{array}$ \\
\hline 4 & $545-565$ & $\begin{array}{l}\text { Land/Cloud/Aerosols } \\
\text { Properties }\end{array}$ \\
\hline 5 & $1230-1250$ & $\begin{array}{c}\text { Land/Cloud/Aerosols } \\
\text { Properties }\end{array}$ \\
\hline 6 & $1628-1652$ & $\begin{array}{c}\text { Land/Cloud/Aerosols } \\
\text { Properties }\end{array}$ \\
\hline 7 & $2105-2155$ & $\begin{array}{c}\text { Land/Cloud/Aerosols } \\
\text { Properties }\end{array}$ \\
\hline 8 & $405-420$ & $\begin{array}{c}\text { Ocean Color/ } \\
\text { Phytoplankton/ } \\
\text { Biogeochemistry }\end{array}$ \\
\hline$\overline{9}$ & $438-448$ & $\begin{array}{c}\text { Ocean Color/ } \\
\text { Phytoplankton/ } \\
\text { Biogeochemistry }\end{array}$ \\
\hline 10 & $483-493$ & $\begin{array}{c}\text { Ocean Color/ } \\
\text { Phytoplankton/ } \\
\text { Biogeochemistry }\end{array}$ \\
\hline 11 & $526-536$ & $\begin{array}{l}\text { Ocean Color/ } \\
\text { Phytopianktoni } \\
\text { Biogeochemistry }\end{array}$ \\
\hline 12 & $546-556$ & $\begin{array}{c}\text { Ocean Color/ } \\
\text { Phytoplankton/ } \\
\text { Biogeochemistry }\end{array}$ \\
\hline 13 & $662-672$ & $\begin{array}{c}\text { Ocean Color/ } \\
\text { Phytoplankton/ } \\
\text { Biogeochemistry }\end{array}$ \\
\hline 14 & $673-683$ & $\begin{array}{c}\text { Ocean Color/ } \\
\text { Phytoplankton } \\
\text { Biogeochemistry }\end{array}$ \\
\hline 15 & $743-753$ & $\begin{array}{c}\text { Ocean Color/ } \\
\text { Phytoplankton/ } \\
\text { Bicgeochemistry }\end{array}$ \\
\hline 16 & $862-877$ & $\begin{array}{c}\text { Ocean Color/ } \\
\text { Phytoplankton' } \\
\text { Biogeochemistry }\end{array}$ \\
\hline 17 & $890-920$ & $\begin{array}{l}\text { Atmospheric } \\
\text { Water Vapor }\end{array}$ \\
\hline 18 & $9 \overline{31-941}$ & $\begin{array}{l}\text { Armospheric } \\
\text { Water Vapor }\end{array}$ \\
\hline 19 & $915-965$ & $\begin{array}{l}\text { Atmospheric } \\
\text { Warer Vapor }\end{array}$ \\
\hline 20 & $3.660-3.840$ & $\begin{array}{c}\text { Surface/Cloud } \\
\text { Temperature }\end{array}$ \\
\hline 21 & $3.92 \overline{9}-3.989$ & $\begin{array}{c}\text { Surface/Cloud } \\
\text { Temperature }\end{array}$ \\
\hline 22 & $3.929-3.989$ & $\begin{array}{l}\text { Surface/Cloud } \\
\text { Teraperature }\end{array}$ \\
\hline 23 & $4.020-4.080$ & $\begin{array}{l}\text { Surface/Cloud } \\
\text { Temperature }\end{array}$ \\
\hline 24 & $4.433-4.498$ & $\begin{array}{l}\text { Atmospheric } \\
\text { Temperature }\end{array}$ \\
\hline 25 & $4.482-4.549$ & $\begin{array}{l}\text { Atmospheric } \\
\text { Temperaure }\end{array}$ \\
\hline 26 & $\Gamma .360^{\circ}=1.390^{\circ}$ & $\begin{array}{l}\text { Cirrus Cloüds } \\
\text { Water Vapor }\end{array}$ \\
\hline 27 & $6.535-6.895$ & $\begin{array}{l}\text { Cimus Clouds } \\
\text { Water Vapor }\end{array}$ \\
\hline 28 & $7.175-7.475$ & $\begin{array}{l}\text { Cirrus Clouds } \\
\text { Water Vapor }\end{array}$ \\
\hline 29 & $8.400-8.700$ & Cloud Properties \\
\hline 30 & $9.580-9.880$ & Ozone \\
\hline 31 & $10.780-11.280$ & Surface/Cloud Temperature \\
\hline 32 & $11.770-12.270$ & Surface/Cloud Temperature \\
\hline 33 & $13.185-13.485$ & Cloud Top Altitude \\
\hline 34 & $13.485-13.785$ & Cloud Top Altitude \\
\hline 35 & $13.785-14.085$ & Cloud Top Altitude \\
\hline 36 & $14.085-14.385$ & Cloud Top Altirude \\
\hline
\end{tabular}

